\title{
VOLUNTADES ANTICIPADAS: DERECHO A DECIDIR
}

\section{ANTICIPATED WILL: RIGHT TO CHOOSE}

\author{
Randall Jiménez Retana ${ }^{1}$ \\ Universidad de Costa Rica \\ ranjim77@gmail.com
}

Publicado 2016

\begin{abstract}
Resumen
Nuestro problema de investigación nace de la preocupación por la carencia de un instrumento de voluntades anticipadas en el sistema de salud y legislación costarricense. Dicha laguna coloca a las personas, hombres y mujeres, en una situación de vulnerabilidad, al no poder autodeterminarse ante procesos de enfermedad y accidentes incapacitantes. Lo anterior compromete el consentimiento informado y las aspiraciones de dichos sujetos en relación con el proceso del final de la vida.
\end{abstract}

Palabras clave: vulnerabilidad, vida, muerte, voluntades anticipadas.

\section{Summary}

Our investigation purpose stems from the problematic lack of any instrument for a living will in the Costa Rican law and health system. This lack of documentation puts people (men and women) in a vulnerable situation of being unable to self-determine their own lives when faced with a debilitating illness or accident. This inability to self-determine one's own life in these situations compromises the informed consent honouring and the wishes of these people in the final processes of life.

Keywords: vulnerability, life, death, advanced directives.

1 El autor es máster en Bioética, UNA-UCR, doctorando en Sostenibilidad y paz en la era posglobal, de la Universidad de Valencia, España, trabaja en la Universidad de Costa Rica, recinto de Golfito, y es coordinador de la Cátedra Temática: Humanidades en el Pacífico Sur. 
La realidad de cada persona, en el proceso del final de la vida, es compleja. Un rasgo común es que las personas no desean sufrir dolores que pueden ser paliados con los medios de los que dispone la medicina en el siglo XXI. La variedad de preferencias de los sujetos, respecto del acceso a la información sobre la condición de su estado de salud, de las posibilidades de tratamientos (curativos, paliativos), de sus deseos en el final de su vida, de la relación con los operarios de salud, de lo que necesitan del sistema de salud y sus facilidades, es muy diversa.

Los sujetos buscan el consejo oportuno de sus operarios en salud, como sujetos garantes de conocimiento respecto de esta y la calidad de vida, en una sociedad donde el acceso a la información es cada vez más inmediato. También esperan que la atención brindada considere su estado particular y pueda apoyárseles desde un enfoque de salud inclusivo. La definición de salud de la Organización Mundial de la Salud (OMS) permite la integración de la esfera familiar, laboral, social y cultural. A la vez, favorece su visualización como proceso. Esto considera no solo el estado de salud física, sino la dimensión emocional de la enfermedad. El encuentro entre salud, enfermedad y final de la vida en los sujetos se torna desafiante. Dicha condición coloca a la persona en un escenario vulnerable, ya que esta circunstancia en la que se encuentra es incierta, insegura, terminal.

Este artículo se centra en la temática de voluntades anticipadas (VA). Entendemos por ello, siguiendo la tesis de Margarita Boladeras (2009):

La expresión de los deseos, prioridades o decisiones de una persona formulados de forma anticipada en previsión de que, en un momento dado, la situación de incapacidad en que se encuentre no le permita manifestarlos. Se trata de una forma de poder continuar ejerciendo el derecho a ser respetado con los valores propios y asegurar que este respeto se mantendrá cuando se presente una situación de mayor vulnerabilidad. El otorgamiento se hace mediante documento (p. 154).

Se propone aquí una reflexión acerca de las VA, en relación con el proceso del final de la vida y las implicaciones de este respecto del envejecimiento, consentimiento informado, autonomía, capacidad, competencia, vulnerabilidad, entre otros, cuyo objetivo es construir una propuesta para el sistema de salud costarricense. Puesto que la legislación costarricense no 
regula el tema y, por ende, no reconoce la validez legal de las $\mathrm{VA}^{23}$, es propicio llevar adelante una fundamentación, desde la bioética, de dicho instrumento del bioderecho. Este acercamiento se inscribe en el interés por promover la toma de decisiones en aquellas personas que han vivido situaciones como accidentes incapacitantes y enfermedades en estado comatoso, para evitar disposiciones que contradigan la voluntad de los sujetos afectados.

Este trabajo aborda las VA en relación con el principio de autonomía y sus subcategorías, tales como competencia y capacidad, siguiendo las tesis de Tom Beauchamp y James Childress (2013), así como la de Margarita Boladeras (2009). Nace de la inquietud por situar la discusión respecto de las voluntades anticipadas en torno a los desencuentros, la toma de decisiones y posiciones que no siempre son fáciles.

\section{Principio de Autonomía}

Según Beauchamp y Childress (2013), el individuo autónomo es aquel que realiza actos libremente, en consonancia con su plan de vida y valores. Asimismo, establecen que las personas poseen libertad en el momento de escoger sus preferencias, sin influjos que tiendan a controlar, manipular o coaccionar su decisión o decisiones, y sean sujetos agentes con capacidad de actuar con intencionalidad en sus actos. Este principio establece al menos tres condiciones necesarias para ello, a saber:

2 En muchos países las voluntades anticipadas son una realidad. Al respecto, es en Estados Unidos donde nace esta tradición y práctica. Algunos autores clásicos en esta temática son: Nancy King con su libro Making Sense of Advance Directives (2007); Allen Buchanan y Dan Brook con Decidir por otros (2009); Daniel Callahan ha aportado muchos escritos a la reflexión, a saber: Poner límites (2004); The troubled dream of life (2000); The Roots of Bioethics (2012). También, Tom Beauchamp y Robert Veatch con Ethical Issues in Death and Dying (1996). Desde The Hasting Center son muchos los documentos emanados. Asimismo, The Presidente's Council on Bioethics con Taking Care (Ethical caregiving in our aging society) (2005). Igualmente, David Doukas y William Reichel con Planning for Uncertainty (2007).

3 En España, Margarita Boladeras ha influenciado la reflexión bioética. Uno de sus últimos libros, El Derecho a no sufrir (2009), aborda el tema de las voluntades anticipadas. Respecto de estas y su relación con el derecho y los prestatarios de salud, están los siguientes autores: Cristina López con Testamento Vital y Voluntades del Paciente (2003); Javier Sancho-Caro y Fernando Abellán con Instrucciones previas en España (2008); Ana Berrocal y José Abellán con Autonomía, libertad y testamentos vitales (2009); Magdalena Palomares y Javier López con El consentimiento informado en la práctica médica y el testamento vital (2002); Federico Montalvo con Muerte digna y Constitución (2009). En Perú, Jessica de Vettori con La autotutela como una nueva institución de amparo familiar (2013). En Uruguay, Gabriela Hormaizteguy con Directivas anticipadas: disposiciones en previsión de la propia discapacidad (2012). 
1. Intencionalidad: las acciones tienen que corresponder con un plan de acción de la persona.

2. Comprensión: debe ser adecuada a cada situación y contexto. Algunas condiciones que la limitan son: enfermedades, irracionalidad e inmadurez.

3. Libertad de acción: sin influencias externas o controles que limiten la libre voluntariedad en la escogencia de oportunidades (Beauchamp y Childress, 2013, pp. 104-105).

La autonomía implica también: “...en algunos contextos, reforzar o mantener las capacidades de otros para la escogencia autónoma mientras se manejan los miedos y otras condiciones que interrumpen o destruyen la acción autónoma" (Beauchamp y Childress, 2013, p. 107). La valoración de las decisiones autónomas, desde el respeto y la consideración, requiere tomar en cuenta todo aquello que pueda afectar a la persona y su mundo valorativo. La desestimación, el ignorar o insultar las voluntades de otros, es una acción que irrespeta la autonomía y el proyecto de vida de los demás.

En la vivencia del sujeto autónomo es necesario considerar algunas reglas, a la hora de entablar relaciones entre usuarios y operarios de la salud, entre ellas:
a. Decir la verdad.
b. Respetar la privacidad de los otros.
c. Proteger la información confidencial.
d. Obtener el consentimiento para intervenciones con pacientes.
f. Cuando pregunten, ayudar a otros a realizar importantes decisiones (Beauchamp y Childress, 2013, p. 107).

La capacidad que posee una persona para decidir de forma autónoma debe precisarse y esclarecerse, ya que no es un concepto unívoco. Esta determinación es relativa en cada situación y puede entrar en conflicto, por su correspondencia o no, con el criterio de los prestatarios de los servicios de salud (Beauchamp y Childress, 2013). Es necesario, por ello, que se deje constancia en un protocolo de VA. 


\section{Principio de vulnerabilidad ${ }^{4}$}

Para Miguel Kottow $(2014)^{5}$, la vulnerabilidad es la condición humana de fragilidad que todos compartimos. En este mismo sentido, cuando la persona se encuentra en el proceso del final de la vida no necesita que se le hable de la muerte, porque ya vive la espera, sino que requiere la ayuda para vivir mejor, sin dolor, reduciendo la sintomatología del sufrimiento. En la propia condición de sujeto vulnerable, es en la cual el sujeto autónomo logra apropiarse de su proceso de salud, gracias a las políticas públicas y a los instrumentos jurídicos que el sistema de salud dispone y ofrece, para la promoción integral de la persona. De esta manera, se propicia una integración entre los límites propios de todo ser humano en el proceso del final de la vida y sus dimensiones tanto personales como familiares.

La finitud humana, las nociones de extraños morales y sociedad pluralistas logran situar la reflexión en torno a conceptos modernos como cuidado, responsabilidad y empatía con los otros. Lo anterior, debido a que el sujeto es frágil, porque tiene que vivir con la mortalidad y con situaciones que lo colocan en riesgo.

Por lo anterior, es que el reconocimiento de la fragilidad humana y del poder de autodeterminación del sujeto no son excluyentes, sino que la promoción de políticas públicas en salud, así como del uso de instrumentos del bioderecho, permitirán brindar posibilidades que expandan la seguridad, reduzcan el dolor y sufrimiento, así como fomentar una sociedad que discierna y discuta sus dilemas éticos, entre otros.

Vulnerabilidad y autonomía tienen por contraparte, al final de la vida, el dolor y sufrimiento que genera una enfermedad, al igual que la toma de decisiones en tales contextos. En Costa Rica, la calidad de vida ha sido asociada a la ausencia de dolor. La búsqueda de la felicidad, para la mayor cantidad de personas, puede favorecerse evitando el dolor. La imagen que aporta el pensamiento clásico y moderno, de que el sujeto es un huésped de la vida, requiere ser más explorada y compartida. En palabras de Alcoberro

4 Véase Rendtorff, J. y Kemp, P. (2000). Basic ethical principles in european bioethics and biolaw (Volumen I: Autonomy, dignity, integrity and vulnerability). Barcelona: Instituto Borja de Bioética, 45-56.

5 Véase, del mismo autor, Kottow, M. (2014). El pa[de]ciente. Una medicina cuestionada. Un testimonio. Santiago, Chile: Ocholibros; Carne y cuerpo. Un desafío a la bioética. Santiago, Chile: Ocholibros. Asimismo, Kottow, M. y Bustos, R. (2010). Antropología médica. Santiago, Chile: Mediterráneo. 
(2013) diríamos que "lo propio de un huésped sensato es saber, también, cuándo debe partir” (p. 195).

\section{La figura jurídica de las voluntades anticipadas ${ }^{67}$}

Este concepto, aunque no está tipificado en la legislación costarricense, sí ha sido implementado en otros países y Estados. California fue "el primer estado que estableció la llamada Ley de la muerte natural ("Natural Death Act”) que entró en vigor el 1 de enero de 1976" (Siurana, 2005, p. 127). Esta legislación instituyó ciertas ideas por resaltar:

- Las personas adultas tienen el derecho de controlar las decisiones relativas a su propio cuidado médico, incluyendo la decisión de no utilizar o interrumpir procesos para mantener la vida en casos de enfermedad terminal.

- La prolongación de la vida por medios artificiales puede causar la pérdida de la dignidad del paciente y dolores y sufrimientos innecesarios.

- En consideración a la dignidad de los pacientes, las leyes del Estado de California reconocerán el derecho de una persona adulta a hacer una declaración por escrito dando instrucciones a su médico sobre la no aplicación o la retirada de procedimientos que pueden mantener su vida en el caso de una enfermedad terminal (Marlasca, 2002, p. 231).

El Consejo de Europa (1997), en el Convenio sobre Derechos Humanos y Biomedicina (Convenio de Oviedo), ha ido estableciendo "normativamente la posibilidad de que los ciudadanos, enfermos o no, otorguen un documento de voluntades anticipadas mediante el cual establecen previsiones de futuro acerca de qué tipo de asistencia sanitaria desean o no desean recibir para el caso en que

6 La teoría del "parens patriae" es de origen angloamericano y hace referencia al "poder soberano de guarda que posee el Estado sobre los individuos que son legalmente incapaces de actuar por sí mismos" (Simón, 2004, p. 21). Sau primera formulación se encuentra en Inglaterra en 1324, bajo el reinado de Eduardo II. El objetivo era que el rey, el cual delegaba las funciones en su canciller, realizara acciones fiduciarias sobre los bienes del afectado, para que una vez que estuviese recuperado, encontrara todo como si el propio sujeto hubiese estado realizando la toma de decisiones. Para ampliar más véase Simón, P. y Barrio, I. (2004). ¿Quién decidirá por mí? Madrid: Triacastela, en especial, las páginas 15-24.

7 Para lograr una visión general de los casos originarios que desencadenaron la reflexión en torno a las voluntades anticipadas y designación de un representante o sustituto, véase Simón, P. y Barrio, I. (2004). ¿Quién decidirá por mí? Madrid: Triacastela, en especial las páginas 24-59. 
se encuentren en un estado de salud determinado" (Royes, 2007, p. 165). Este paso es una expansión del principio de respeto a las personas. Cada quien ejerce su autonomía, durante todo el período de su vida. La tradición del consentimiento informado favorece que se desarrollen instrumentos de esta naturaleza, que resguarden las voluntades personales en todos sus extremos.

El documento de voluntades anticipadas, de la mano del consentimiento informado, está modificando las relaciones sanitarias (cf. Royes, 2007). El asunto está en determinar cuáles pueden ser los aportes de los documentos de las voluntades anticipadas. Lo anterior debido a que una primera reacción es pedir resultados o beneficios de su implementación en un sistema de salud. La confianza de que las decisiones tomadas con previsión sean consideradas por los operarios de la salud, en el momento de considerar cualquier procedimiento desde los valores y solicitudes de la persona que hoy no es capaz de comunicarlas, favorece la tranquilidad de los sujetos en el final de la vida. El sufrimiento y dolor siempre son aspectos por considerar, así las voluntades anticipadas aminoran estas realidades al ofrecer la posibilidad de disponer previamente lo que corresponde en el caso particular. El acercamiento que plantea Royes (2007) al respecto es esclarecedor para considerar las voluntades de las personas en todo el proceso del final de la vida:

Deben entenderse como un consentimiento informado realizado con anterioridad al supuesto o supuestos en los que se debería entrar en vigor (sería un No consentimiento, ya que lo que se solicita es que no actúe de determinada manera en circunstancias precisas). El sujeto capaz y competente determina las condiciones bajo las cuales no quiere recibir ciertas terapias o procedimientos médicos que alarguen el proceso de la vida en detrimento de su dignidad individual (encarnizamiento terapéutico) (pp. 166-167).

La limitación del esfuerzo terapéutico (LET) en la práctica clínica es frecuente por lo que, mediante un documento de voluntades anticipadas, el paciente participa de modo directo en este tipo de decisiones desde el momento en que su voluntad puede ser conocida por el personal sanitario que le atiende, aunque él, personalmente, no esté ahora en condiciones de manifestarla: ya lo ha hecho antes y su voluntad debe considerarse prioritaria ante la de otros. Facilita una relación asistencial basada en la colaboración y dirigida a conseguir un objetivo común a médicos y pacientes: la promoción 
y la protección de los intereses del paciente de acuerdo con lo que este entiende como sus intereses en el ámbito de la asistencia sanitaria, es decir, en consonancia con sus prioridades y opciones personales, con su visión de la vida y la muerte.

Existe una corriente, cada vez más grande, que reconoce la autonomía del sujeto en todos sus extremos, por lo que la previsión y disposición en diversas situaciones deben ser consideradas en una legislación y en el sistema sanitario en particular. Por ello, a nivel internacional se ha implementado modelos de acreditación que favorecen las consideraciones de las voluntades de los pacientes como un asunto primordial en la toma de decisiones.

Por lo anterior, Simón (2005) en el modelo de acreditación del Health Quality Service (HQS) señala que: "sobresalen las necesidades individuales y derechos del paciente, resaltando lo referente a la autonomía, la información y el consentimiento informado... Atención a los pacientes terminales, moribundos..." (p. 78).

Este sistema de acreditación, en su sección 3. ․ , establece que la experiencia del paciente dispone de tres normas, de las cuales son pertinentes para nuestro trabajo la Norma 21: Los derechos del paciente y la Norma 22: Las necesidades individuales del paciente.

La HQS establece en sus protocolos la evaluación de la capacidad y decisiones de representación -incluyendo el uso de directivas anticipadas-: 21.7, 21.19, 22.16, 25-10, 27.5.10. Como parte de los derechos explícitamente reconocidos por la HQS, está el derecho a que se respeten sus últimas voluntades (voluntades anticipadas): 22.16, a saber:

Existe un procedimiento documentado y escrito/revisado en los últimos tres años acerca de la respuesta de la organización para dar cumplimiento a las últimas voluntades de pacientes/usuarios (A-ISO).

Orientación: este procedimiento necesita la formación del personal acerca del status legal y ético de las últimas voluntades (por ejemplo, deben hacerse cuando el paciente/usuario tenga capacidad de dar consentimiento válido), cómo el personal es consciente de ellos, cómo se custodian (por ejemplo, debería haber una anotación completa en la historia clínica acerca de cualesquiera últimas voluntades) y cómo se llevan a cabo (Simón, 2005, p. 139) 
La práctica de dejar constancia de los deseos personales en torno al proceso de la salud y la enfermedad es considerada por muchas instancias para ser evaluada, para el otorgamiento de acreditaciones en el nivel internacional. Lo anterior, para certificar la calidad de la prestación de servicios por la institución hospitalaria, así como por los profesionales involucrados en todo el proceso. Con el desarrollo de la teoría del consentimiento informado se abre el espacio propicio para la consideración de un área más amplia respecto a la autonomía del usuario en salud. Siurana (2005) presenta algunos países que han ido incorporando a sus sistemas de salud estas disposiciones, a saber:

a. Estados Unidos: Ley Federal de Autodeterminación del Paciente (Patient Self Determination Act). Aprobada por el Congreso de Estados Unidos en 1990 y puesta en vigor el 1 de diciembre de 1991.

b. Australia: en el Estado de Victoria es promulgada la Ley del Tratamiento Médico de Victoria en 1988 (Victorian Medcal Treatment Act 1988). En otro Estado como Australia del Sur se promulga la Ley del Consentimiento al Tratamiento Médico y Cuidados Paliativos en 1995 (Consent to Medical Treatment and Palliative Care Act 1995)

c. Canadá: son varias las provincias que promulgan diversas leyes para legalizar las voluntades anticipadas (advance directives): Alberta, Nueva Foundland, Manitoba, Ontario, Québec, Nueva Escocia.

d. Dinamarca: el concepto utilizado es el de testamentos vitales (Livstestamente), y es regulado por la Ley $\mathrm{N}^{\circ} 482$ de 1998. En la orden $\mathrm{N}^{\circ}$ 663 del 14 de setiembre de 1998 se regulan las disposiciones respecto de los médicos y los testamentos vitales.

e. Holanda: en la Ley de Tratamiento Médico de 1995 se reconocen las voluntades anticipadas.

f. Argentina: durante la XXII Jornada Notarial Argentina del año 1992 el señor G.A. Rubio pone a consideración un documento llamado "Testamento para la vida". En su Código Civil (arts. 59 y 494) se establece el "Asesor de menores incapaces" (cf. López, 2003, p. 27-48).

La dimensión familiar es determinante de cómo la persona va asumiendo y viviendo su proceso del final de la vida. Por ello, este momento es individual, único e intransferible. Para Boladeras (2013) se requiere de un "acompañamiento pertinente de las personas que la rodean" (p. 214). 
Esta dimensión de compañía implica, a la vez, un reconocimiento del proceso completo: vida y muerte. Por lo que "no hay muerte propiamente humana sin el reconocimiento, por parte de los demás, del carácter único e insustituible de esa vida que se extingue. Podemos decir que la vida es sagrada en el sentido de considerarla merecedora de dignidad y de respeto; la muerte cierra el círculo de la vida y es tan sagrada como ésta" (Boladeras, 2013, p. 214). El ocultamiento de una condición de salud, o el estado de progreso de una enfermedad, es necesario que sea de conocimiento por parte del usuario de los servicios de salud. Reconocer a la persona es tratarla como competente y capaz de decidir sobre sus propias aspiraciones.

La sociedad contemporánea no escapa a los excesos en la extralimitación del esfuerzo terapéutico. Algunas consecuencias de ello se ubican en querer medicalizarlo todo, y procurar la hospitalización de la persona que se encuentra en su proceso del final de la vida. La actuación que debe pedirse a las instituciones, en especial las prestatarias de servicios de salud, es la de regirse "con justicia en todas sus actividades" (Boladeras, 2013, p. 215).

\section{Toma de decisiones}

La autonomía de la persona en un proceso del final de la vida puede darse en las situaciones del presente, pero también de manera prospectiva. Esta característica es fundamental en el proceso del final de la vida. La toma de decisiones en este proceso reviste un carácter ético.

Una persona, según Siurana (2005), realiza acciones autónomas cuando "el que actúa lo hace: intencionalmente, con comprensión y sin coacción, es decir, sin influencias controladoras que determinen su acción” (p. 42). Por tanto, para ajustar las acciones de los sujetos, debe darse necesariamente el discernimiento ético. Las personas deben estar sin presión ni coacción, ser capaces de tomar sus propias decisiones, identificando las consecuencias que se derivan de estas y sus implicaciones. Sin tal deliberación, la bioética se ve vaciada de todo contenido. Bioética es diálogo pluralista, deliberación, consensos. Solo cuando la persona es respetada en lo que ha decidido y sus puntos de vista, de acuerdo con sus valores y creencias, estamos frente a un grupo social que reconoce a los sujetos como seres autónomos. La concreción de lo anterior es el consentimiento informado en la bioética. 
Este discernimiento, diálogo ético, está mediado por las metas de la medicina propuestas por el Hasting Center (1996), el cual en su informe establece cuatro metas claramente identificables:

a) La prevención de la enfermedad y de las lesiones, y la promoción y mantenimiento de la salud.

b) El alivio del dolor y el sufrimiento causado por la enfermedad y las dolencias.

c) La asistencia y curación de los enfermos y el cuidado de los que no pueden cuidarse.

d) Evitar la muerte prematura y velar por una muerte en paz.

Los sujetos pueden manifestar sus voluntades anticipadas de manera oral o escrita. Esta última se recomienda, preferentemente, para evitar contratiempos en los momentos determinantes, cuando es necesario reconocer lo que el sujeto expresó respecto de cuidados de la salud y disposiciones para después de la muerte, en materia de disposición de su cuerpo (donación de órganos, investigación). Las voluntades anticipadas adoptan varias formas ante situaciones en el proceso del final de la vida, a saber:

a) Instrucciones previas: sobre los tratamientos que desea o no; sobre la disposición de su cuerpo una vez acontecida la muerte.

b) Designación de representante: para cuando el sujeto no sea capaz y competente para autodeterminarse, o para que, después de la muerte, disponga de su cuerpo y órganos según su voluntad, valores y costumbres.

c) Historia de valores: cuáles son la cosmovisión, deseos, valores y actitudes que tienen que considerarse ante eventuales tratamientos y decisiones que deban tomarse.

Según Sánchez-Caro y Abellán (2008), en el proceso del final de la vida no siempre es fácil y claro determinar las voluntades y deseos de los sujetos involucrados. Estos autores señalan algunas de las "dificultades que en las decisiones al final de la vida supone la asimetría informativa entre los profesionales sanitarios y los pacientes y la influencia que tienen los familiares en la toma de decisiones" (p. 23). Estas características generan espacios propicios para situar a esta población (adultos mayores y sujetos en 
el proceso del final de la vida) en condiciones de vulnerabilidad (Beauchamp y Childres, 2013).

En este proceso hacia la muerte, el abstenerse de ciertas prácticas terapéuticas aleja al ser humano de procedimientos que pueden dar como resultado el encarnizamiento terapéutico y tratamientos fútiles o inútiles. Aunque la consecuencia sea positiva, no necesariamente es recomendable. El costo del procedimiento, emocional, físico, económico, y su impacto en la calidad de vida del sujeto deben considerarse con sumo cuidado. Cuando la salud ya no es posible y los indicadores vitales no son alcanzables, las decisiones médicas tienen que sopesarse desde los operarios de salud, comités de bioética, voluntad del sujeto, o de su representante, si fuera el caso. Dependerá también de qué clase de sistema de salud esté involucrado: público o privado. Todo este universo de posibilidades impacta las decisiones finales.

El Hasting Center (2004) plantea como un fin de la medicina "la búsqueda de una muerte tranquila”. Este centro señala al respecto:

Dado que la muerte nos llega a todos (los pacientes de todo médico habrán de morir algún día, al igual que el propio médico), la medicina ha de considerar una prioridad la creación de unas circunstancias clínicas que favorezcan una muerte tranquila. Una muerte tranquila puede definirse como una en la que el dolor y el sufrimiento se reduzcan mediante unos cuidados paliativos adecuados, en la que al paciente nunca se le abandone ni descuide y en la que los cuidados se consideren igual de importantes para los que no vayan a sobrevivir como para los que sí. Por supuesto que la medicina no puede garantizar una muerte tranquila ni responsabilizarse por lo que las personas traen consigo a su lecho de muerte. Pero lo que sí puede evitar la medicina es considerar la muerte como un accidente biológico evitable o un fracaso médico. Más tarde o más temprano, la muerte es, como ha sido siempre, el resultado inevitable incluso del mejor tratamiento médico. En toda vida humana llegará un momento en que un tratamiento de soporte vital será inútil; se llegará al límite absoluto de las capacidades de la medicina. Por tanto, la gestión humanitaria de la muerte es la responsabilidad final, y probablemente la más exigente desde el punto de vista humano, del médico, que está obligado a reconocer en su paciente tanto su propio destino como las limitaciones inherentes a la ciencia y arte de la medicina, cuyos objetos son seres mortales, no inmortales (pp. 46-47). 
Esto favorece la esperanza de una formación académica rigurosa en los profesionales de la salud, para que puedan, de manera habitual, ser capaces de "tratar el dolor de sus enfermos a fin de no tenerlos que derivar a centros especializados de manera generalizada" (Boladeras, 2013, p. 222). En zonas geográficas como las costarricenses, donde el transporte y desplazamiento de sujetos, en condiciones delicadas de salud, se vuelve complicado y comprometedor, es urgente se discuta este tipo de formación.

Las VA permiten que los sujetos autónomos puedan tomar decisiones con previsión, pero requieren que se sensibilice a los operarios de la salud. El respeto por la autonomía personal, la toma de decisiones desde los valores y cosmovisiones personales, se torna fundamental. Margarita Boladeras lo expresa con claridad:

Asumir la enfermedad, el dolor y la muerte desde la propia vida implica poder tomar decisiones de acuerdo con la trayectoria vital de cada ser humano. El respeto a la autonomía es por lo tanto fundamental. Seguir o no un tratamiento, suspender un procedimiento, aceptar o no la hospitalización, etcétera son decisiones que dependen del enfermo y se debe velar para que así sea (2013, pp. 226-227).

La consideración de los objetivos esenciales, para los que fueron creados los servicios de salud, debe ser propuesta en los tiempos actuales: comunicación desde los intereses y necesidades de los pacientes; el contacto mediante gestos, presencia, actitudes y complicidades. Todo lo anterior para que sea "una presencia que acompañe y tranquilice" (Boladeras, 2013, p. 229).

\section{Conclusiones}

¿Los documentos de las VA aportan la respuesta perfecta a los dilemas en el proceso del final de la vida? No. Sin embargo, representan un esfuerzo por alcanzar niveles de autodeterminación en los pa(de) cientes $^{8}$. La capacidad y la competencia de las personas no deberían subestimarse ante la posibilidad de facilitar mejores derroteros para la toma de decisiones anticipadamente.

8 Véase Kottow, M. (2014). El pa[de]ciente. Una medicina cuestionada. Un testimonio. Santiago, Chile: Ocholibros. 
Por ello, el debate en Costa Rica sobre esta temática, que aún es nuevo, está enmarcado, en no pocas ocasiones, desde discursos que no logran fomentar el diálogo y toma de decisiones, sea por posturas centradas en una visión absolutista y sagrada de la naturaleza humana o por corrientes que pretenden hacer realidad el mandamiento tecnocientífico. Esto hace infructífera cualquier iniciativa, ya que se descarta por sus proponentes y no por su fundamento.

Sin embargo, el sistema de salud costarricense tiene en sus clínicas del dolor y cuidados paliativos un espacio de esperanza para iniciar un proceso de sensibilización respecto de las VA. Los equipos de trabajo multidisciplinario en dichas clínicas favorecen tal proceso de las personas y sus familiares. Sin embargo, se requiere de un esfuerzo legislativo para otorgar seguridad jurídica a los operarios de la salud, como a las familias del usuario.

Ante la carencia de un instrumento de VA, se silencia al pa(de)ciente. El esfuerzo por lograr mayores niveles de dignidad en las personas tiene que considerar todos los estadios del desarrollo en los sujetos. Por ello, avanzar en la discusión de las VA implicará un diálogo abierto, heterodoxo, incluyente, humanizador. La meta: una buena muerte, desde los fines de cada persona.

\section{Bibliografía}

Beauchamp, T. y Childress, J. (2013). Principles of Biomedical Ethics (séptima edición). New York: Oxford University Press.

Boladeras, M. (2009). Derecho a no sufrir. Barcelona: Los libros del lince.

. (2013). El impacto de la tecnociencia en el mundo humano: Diálogos sobre bioética.

Madrid: Tecnos.

López, C. (2003). Testamento vital y voluntad del paciente. Madrid: Dykinson.

Marlasca, A. (2002). Introducción a la Bioética. Heredia: Facultad de Filosofía y Letras, Universidad Nacional.

Royes, A. (2007). El consentimiento informado y los Documentos de voluntades anticipadas. En Casado, M., Nuevos Materiales de Bioética y Derecho (pp. 159-176). México: Fontamara.

Sánchez, J. y Abellán, F. (2008). Instrucciones previas en España. Aspectos Bioéticos, jurídicos y prácticos. Granada: Comares.

Simón, P. (2005). Ética de las organizaciones sanitarias. Nuevos modelos de calidad. Madrid: Triacastela.

Siurana, J. (2005). Voluntades anticipadas. Una alternativa a la muerte solitaria. Madrid: Trotta. The Hasting Center. (1996). The Goals of Medicine. Setting New priorities: Hasting Center Report 26/6, suplemento especial, pp. S1-S27. 